\title{
Features of the Cosmic Ray Anisotropy in the Minima for \# 23 - 25 Solar Cycles
}

\section{Krzysztof Iskra}

Polish Air Force University

Deblin, Poland

E-mail: k.iskra@law.mil.pl

\section{Witold Wozniak}

Polish Gas Company

Warsaw, Poland

\section{Marek Siluszyk}

Siedlce University

Siedlce, Poland

\section{Renata Modzelewska}

Siedlce University

Siedlce, Poland

\section{Michael Alania}

Siedlce University

Siedlce, Poland

\begin{abstract}
We study the role of drift effect in the temporal changes of the anisotropy of galactic cosmic ray (GCR) and the influence of the sector structure of the heliospheric magnetic field (HMF) on it. We analyze the GCR anisotropy in the solar cycle 24 and solar minima: 23/24 with negative polarity $(A<0)$ for the period of 2007 - 2009 and 24/25 with positive polarity $(A>0)$ in $2017-2018$ using data of global network of Neutron Monitors (NMs). We use the harmonic analyses method to calculate the radial $A_{r}$ and tangential $A_{f}$ components of the anisotropy of GCR for different sectors ('+' corresponds to the positive and '-' to the negative directions) of the HMF. We compare the analysis of the GCR anisotropy using the median rigidity $\mathrm{R}_{\mathrm{m}}$ of NM response (e.g., Ahluwalia et al. 2015) and the effective rigidity $\mathrm{R}_{\mathrm{ef}}$ characteristic for each NM proposed by Gil et al. (2017). Then the radial $A_{r}$ and tangential $A_{f}$ components are used for characterizing the GCR modulation in the heliosphere. We show that in the solar minimum 23/24 in 2007-2009 when $A<0$, the drift effect is not visibly evident in the changes of the component, i.e. for this solar minimum drift effect is $\sim 4 \%$.
\end{abstract}

36th International Cosmic Ray Conference -ICRC2019-

July 24th - August 1st, 2019

Madison, WI, U.S.A. 


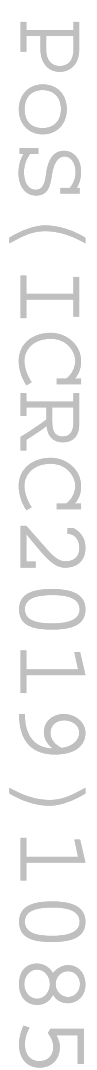

